\title{
New Therapies for Chronic Hepatitis C Virus Infection
}

\author{
Anouk Dev, MD, Keyur Patel, MD, and John G. McHutchison, MD
}

\author{
Address \\ Duke Clinical Research Institute, Duke University Medical Center, \\ PO Box 17969, Durham, NC 277I5, USA. \\ E-mail: mchut00I@mc.duke.edu \\ Current Gastroenterology Reports 2004, 6:77-86 \\ Current Science Inc. ISSN 1522-8037 \\ Copyright () 2004 by Current Science Inc.
}

Chronic hepatitis $C$ infection is associated with significant morbidity and mortality in addition to substantial social and health-related costs. Since the identification of the virus and determination of the HCV genome over a decade ago, considerable progress has been made in the treatment of chronic hepatitis $C$ infection. However, the current standard combination of interferon-based therapies and ribavirin is effective in only $50 \%$ of patients. In addition, this combination is expensive, requires lengthy periods of administration, and is associated with significant side effects. Furthermore, no effective preventive measure, such as vaccination, is currently available. A number of newer therapies, including protease and helicase inhibitors, ribozymes, antisense therapies, and therapeutic vaccines, are in preclinical and clinical development and may significantly enhance existing therapeutic options for the future.

\section{Introduction}

Hepatitis $\mathrm{C}$ virus (HCV) infection represents a viral pandemic affecting approximately 170 million chronically infected people [1]. Worldwide, HCV infection is more prevalent than the hepatitis $B$ virus and HIV-1 infections, and more than 1 million new cases are reported annually [2]. Progression to chronic hepatitis C (CHC) occurs in the majority of infected individuals. Of those who are chronically infected, $5 \%$ to $20 \%$ will develop liver cirrhosis over a 10 - to 20 -year period, and $2 \%$ to $5 \%$ of patients with cirrhosis due to HCV will develop hepatocellular carcinoma or other liver-related complications annually $[3,4]$. In most countries where data are available, HCV infection is the leading cause of liver transplantation [5]. In the absence of a universally effective vaccine to prevent new infections, antiviral therapy represents the best prospect of controlling viral replication and preventing significant hepatic disease. In this article we provide a broad overview of potential emerg- ing therapies for this common infection. Compounds now in clinical development are summarized in Table 1.

\section{Molecular Virology of Hepatitis C Virus Infection}

Hepatitis C is an enveloped, single-stranded, 9.5-kb RNA virus that encodes a single polyprotein of 3011 amino acids. The distinct nucleotide sequence of the HCV genome gives rise to specific genotypes and subtypes [6]. This virus is post-translationally cleaved into 10 proteins in the structural region by a host signal peptidase and into regulatory proteins in the nonstructural regions by HCV-encoded proteases. The structural region contains the core and two envelope proteins (E1 and E2). The nonstructural proteins support viral RNA replication and function as proteases (NS2, NS3, and NS4A), helicases (NS3), and an RNAdependent RNA polymerase (NS5B) $[7,8]$. HCV also encodes two highly conserved untranslated regions (UTR) at either end of the virus - the 5'UTR and 3'UTR. The 5'UTR contains an internal ribosome entry site (IRES), which serves as the initiation point for translation [9]. These RNA structures and viral proteins play a pivotal role in the HCV life cycle. The virus particle attaches to the host cell and enters via low-density lipoprotein receptors and possibly coreceptors such as CD81. The virus uncoats to release its single-stranded RNA genome in to the cytoplasm. At the surface of the endoplasmic reticulum (ER), the HCV genome is translated and processed by a combination of cellular and virus-encoded proteinases to form mature proteins. Viral nonstructural and host proteins form a replication complex, which undertakes the replication of the viral RNA, generating more positive strands via a double-stranded intermediate. Viral particles acquire an envelope by budding through the ER membranes. HCV virions pass through the Golgi complex before being exported from the cell (Fig. 1). Viral replication occurs through an RNA-dependent RNA polymerase that lacks a proofreading function, resulting in the rapid evolution of diverse but related quasispecies within an infected individual. Mutations in these nucleotides may interfere with viral protein synthesis. Alternatively, molecular-based therapies targeting these critical structures can also affect viral replication. Given the diversity of nucleotide sequences in the $\mathrm{HCV}$ genome, molecular-based therapies must target a 







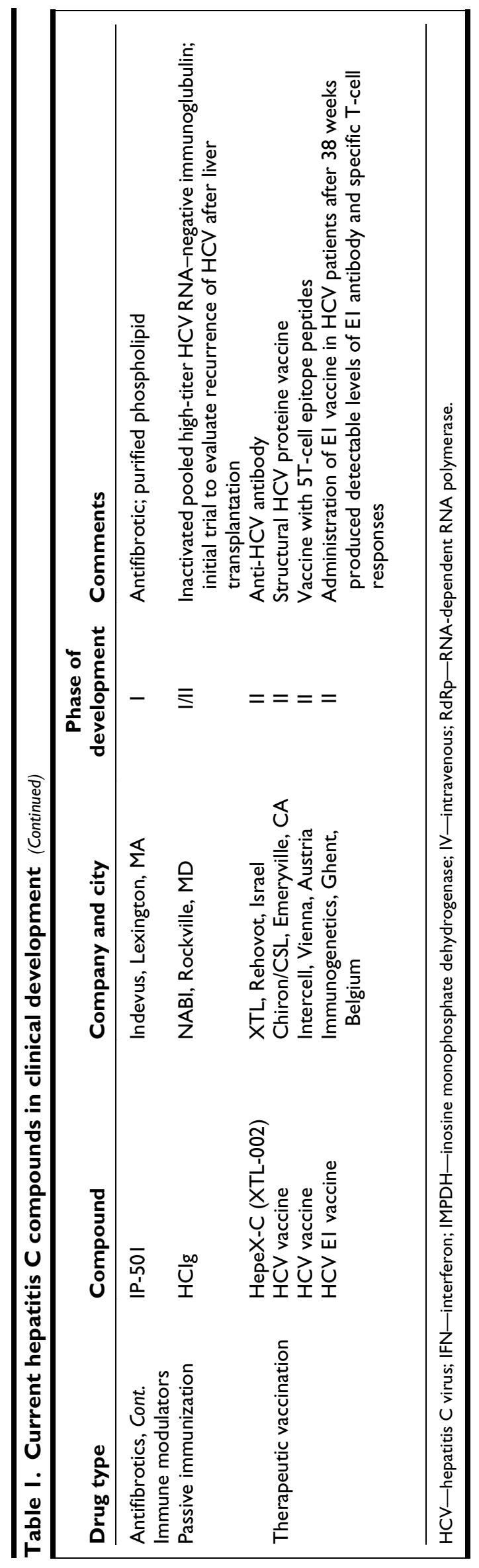




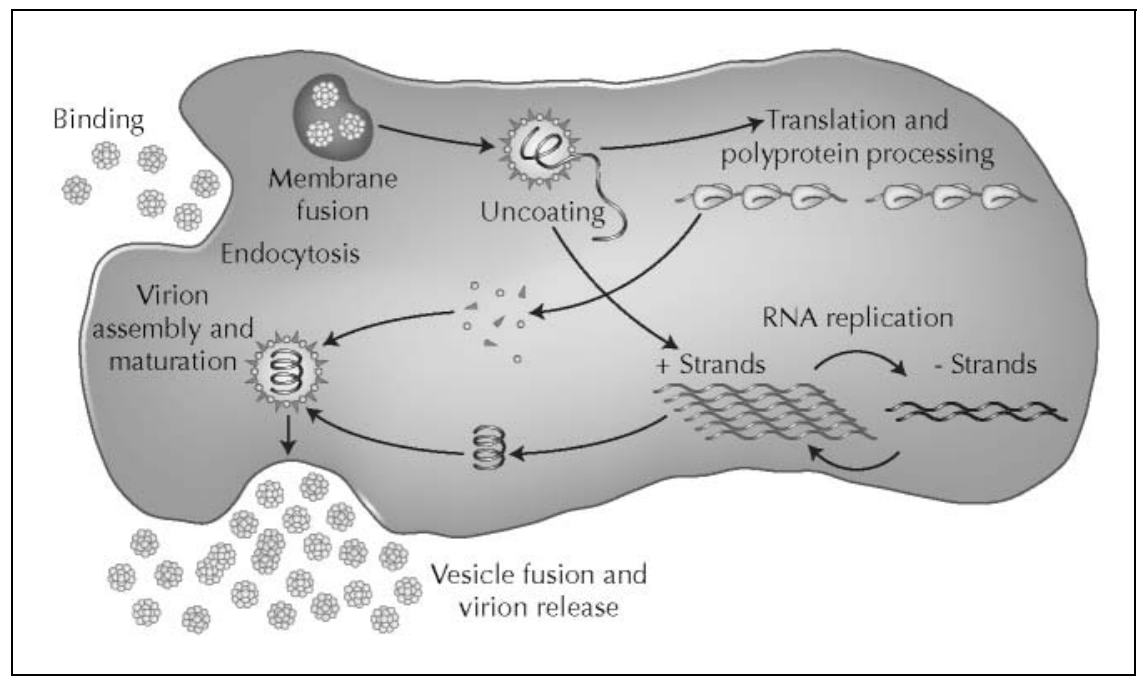

Figure 1. HCV is a positive-sense, single-stranded RNA virus containing a 3000-amino acid encoded polyprotein flanked by a highly conserved 5' and $3^{\prime}$ terminal region. The virion gains access to the host cell by binding to unknown receptor(s) on the hepatocyte cell surface and is transferred into the cytosol by endocytosis. It then uncoats the nucleocapsid, allowing the viral proteins to be translated by the viral genome. The translated polyprotein product is processed by the host signal pepsidases into structural and nonstructural (NS) proteins. During RNA replication, the NS proteins form the replication initiation complex, which initiates the synthesis of negative RNA genomic strands that serve as a template for production of positive RNA strands. The structural core protein with the viral RNA forms the core of the virus particle, which acquires an envelope by budding through the endoplasmic reticulum (ER) membrane. HCV virions pass through the Golgi complex before being exported from the cell. (Adapted from Davis et al. [61].)

variety of viral proteins for maximal efficacy, while preventing or diminishing the development of resistance.

\section{Strategies for Drug Development}

The current standard of treatment consists of interferon (IFN)-based therapies combined with the nucleoside analogue ribavirin. The regimen of standard IFN and ribavirin achieves a sustained virologic response (SVR, defined as undetectable serum hepatitis C RNA 24 weeks after completion of therapy) rate of $38 \%$ to $43 \%[10 \bullet \bullet, 11 \bullet \bullet]$. The addition of a polyethylene glycol moiety to standard IFN (pegylated IFN) reduces drug clearance, thereby allowing weekly dosing, and has further enhanced SVR to greater than $50 \%[12 \bullet \bullet, 13 \bullet \bullet]$. Although standard combination therapy and the introduction of pegylated versions of IFN have led to marked improvements in the treatment of $\mathrm{CHC}$, these regimens are only effective in approximately half of the patients treated. They are also expensive, prolonged (6-12 months), associated with significant side effects, and clearly not suited for all patients $[10 \bullet \bullet-13 \bullet \bullet]$. Current therapy remains inadequate for a significant number of patients who fail to respond and in certain groups of patients, including those with advanced liver disease and those in whom combination therapy is contraindicated. An ideal therapy for CHC would have enhanced efficacy, fewer side effects and contraindications, and be less costly. Alternative or future therapeutic strategies include manipulation of our existing treatments, treatments designed to induce a viral specific host immune response, and therapy to reduce hepatic injury. It is unlikely that a single agent playing such a role will be available in the near future, but a number of therapies in clinical development may improve existing therapeutic options.

\section{Limitations to Drug Development}

Although considerable progress has been made in defining the natural history of HCV infection and understanding its molecular virology, the development of alternative drugs has been limited by the absence of a reliable cellbased experimental system supporting HCV replication and the paucity of effective animal models. A well-established model in which HCV replication has been successful is the chimpanzee, but issues of cost and availability have limited its use [14]. More recently, investigators have described transgenic mouse models with human hepatocyte xenografts that permit HCV replication $[15,16]$. Additionally, human hepatoma cell lines have provided a system whereby HCV nonstructural proteins can replicate, allowing testing of drugs in genomic and subgenomic replicons. Although these models are effective for the study of viral replication, they are incapable of producing infectious virions, thereby limiting the study of infectivity $[17,18]$. Alternative surrogate systems such as bovine virus diarrheal virus, which shares common features with $\mathrm{HCV}$, have been widely used for anti-flavivirus drug discovery, allowing in vitro infectivity testing. However, not all compounds that are active against this model are active against HCV [19]. 


\section{Enhancing Existing Therapies} Interferon-based therapies

Interferons are a group of cytokines that exhibit antiviral, antiproliferative, and immunomodulatory effects. They exert their effects through a series of complex signal pathways, resulting in the expression of IFN inducible genes, which in turn mediate immunoregulatory actions, including activation of macrophages and natural killer (NK) cells and upregulation of Th1 cells. IFNs may also suppress cell proliferation and inhibit virus replication in cells [20]. Although IFNs are the mainstay of current antiviral treatment regimens, the use of these agents is complicated by suboptimal pharmacokinetic profiles and biologic activity, leading to a less than adequate therapeutic response. In an attempt to improve therapeutic efficacy, drug development has focused on modification of existing IFN compounds.

\section{Modified interferon compounds}

Classification of IFNs is based on the distinct cell surface receptor to which they bind. Type I IFNs include IFN- $\alpha,-\beta$, and -omega, and type II IFNs include IFN- $\gamma$. The IFN preparations approved for use in HCV include IFN- $\alpha 2 a$, IFN$\alpha 2 \mathrm{~b}$ subtypes, and consensus IFN (CIFN). In addition to pegylation, naturally occurring IFNs can be modified through primary amino acid sequence alteration. IFN-alfacon 1 is a cytokine that was engineered to contain the specific amino acid sequences found in other IFN- $\alpha$ subtypes. Sustained virologic and biochemical response rates of patients treated with these CIFNs in randomized, controlled trials have varied according to the populations under study. In one study, high-dose CIFN showed superiority over standard IFN in treatment-naïve patients [21]. However, in a comparison of equivalent doses, these IFNs were equally effective [22]. Other IFNs have been produced by "gene shuffling" of IFN- $\alpha$ DNA-encoding sequences, resulting in markedly increased antiviral activity in vitro, though this approach has not yet been tested in humans. Additionally, fusion products, such as IFN and serum albumin, have resulted in molecules with superior pharmacokinetics and pharmacodynamics, compared with standard IFN in vitro $[23,24]$. Other IFN species, such as IFN- $\gamma$ and -omega, have been shown to inhibit protein synthesis and RNA replication of subgenomic and genomic HCV replicons and are also being evaluated in humans [25]. Additional delivery systems designed to improve the pharmacokinetic profile of existing IFNs include disposable infusion pumps, controlled release formulations ( $\mathrm{eg}$, sustained release devices), and oral formulations. The efficacy and safety of these "modified" IFNs in patients with $\mathrm{HCV}$, given alone or combined with ribavirin, need to be established through carefully controlled clinical trials.

\section{Oral interferon inducers}

Oral IFN inducers are compounds that effect an immune response by inducing or modulating the release of host cytokines. Oral agents, including the imidazoquinolones imiquimod and resimiquod and nucleoside analogues ANA245 and ANA971, induce the innate IFN response and are being evaluated in patients with HCV [26]. Imiquimod, currently approved for use as a topical agent in dermatology, has been associated with toxicity in human studies [27], and resiquimod is in phase II evaluation in CHC patients. ANA245 induces numerous cytokines in addition to IFN- $\alpha$ and has shown antiviral activity against a broad range of viruses. However, reduced bioavailability has led to the development of an intravenous form now in early clinical trials. ANA971 is a novel molecule that efficiently delivers ANA245 after oral administration in animal models.

\section{Ribavirin-like drugs and inosine \\ 5 'monophosphate dehydrogenase inhibitors}

Ribavirin monotherapy is ineffective in inducing sustained HCV clearance or reductions in HCV RNA concentrations, but it markedly potentiates the effect of IFN, resulting in superior response rates. Although not clearly understood, several mechanisms of action of ribavirin have been proposed, including immune-mediated activity on the host Th1/Th2 cytokine balance, inhibition of the host inosine monophosphate dehydrogenase (IMPDH: a rate-limiting enzyme in guanine nucleotide synthesis) activity against viral RNA-dependant RNA polymerase, and as an RNA viral mutagen. Investigators have suggested that ribavirin may act as an IFN sensitizing agent, making virus-infected cells more responsive to IFN through the ability of ribavirin to activate IFN signaling pathways [28•]. The use of ribavirin is complicated by the development of hemolytic anemia, which often limits effective dosing, making development of compounds without this toxicity an attractive prospect for combination therapy regimens.

Levovirin is an L-sugar analogue of ribavirin that has similar immunomodulatory action. The degree of IMPDH inhibition by levovirin compared with ribavirin is unclear, but levovirin appears to be associated with a lower incidence of hemolytic anemia [29]. In phase I trials, levovirin was tolerated well, and proof-of-concept studies of this agent in combination with pegylated IFN- $\alpha$ are underway [30]. Viramidine is a carboxamide prodrug that is converted to ribavirin and other phosphorylated metabolites in the liver. The first-pass effect and retention of these metabolites within the liver after oral dosing suggest that less drug is available to other tissues, including erythrocytes. In phase I trials, viramidine was associated with a lower incidence of hemolytic anemia but otherwise had a side-effect profile similar to that of ribavirin [31]. Viramidine is undergoing phase II proof-of-concept trials in combination with pegylated IFN- $\alpha$.

Other IMPDH inhibitors include VX-497, an orally bioavailable agent that has an additive inhibitory effect on viral replication when combined with IFN and ribavirin in the HCV replicon system. VX-497 monotherapy is ineffective in suppressing viral replication in human studies, but in combination with IFN- $\alpha$ it has similar antiviral effects 


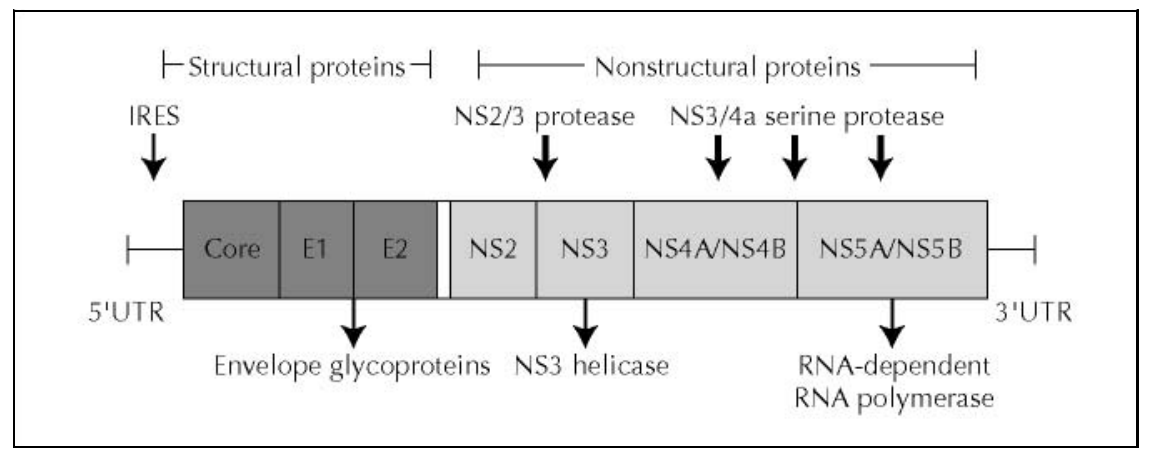

Figure 2. Schematic representation of the hepatitis $\mathrm{C}$ virus (HCV) genome and sites for drug development. The key virus-encoded proteins are highlighted. At the 5 untranslated region, (5'UTR), the internal ribosome entry site (IRES) is where the antisense oligonucleotides and ribozymes are developed. The NS3 region encodes a specific helicase, and the NS5A region encodes an RNA-dependent RNA polymerase. These enzymes, together with the NS2/3 and NS3/4 proteases, are targets for drug development.

after 4 weeks of treatment to those with IFN monotherapy [32]. Therapeutic studies of longer duration involving IFN, ribavirin, and VX-497 are awaited. Mycophenylate mofetil (MMF), approved for use as an immunosuppressant following organ transplantation, is also an IMPDH inhibitor. However, MMF combined with IFN- $\alpha$ was ineffective in improving the virologic response in $\mathrm{CHC}$ patients who had previously not responded to standard therapy, suggesting that mechanisms other than IMPDH inhibition are important in suppressing viral replication [33]. MMF is being evaluated in combination with pegylated IFN in current clinical trials [34].

\section{Other immunomodulatory drugs}

Thymosin- $\alpha 1$

Thymosin- $\alpha 1$ is a synthetic nonglycosylated peptide derived from thymus gland extracts. This drug stimulates the production of cytokines, including IFN- $\gamma$ and interleukins (IL)-2 and -3, and promotes T-cell maturation and NK activity. In combination with IFN- $\alpha$, it is tolerated well; however, available data do not allow adequate interpretation of the antiviral effect or virologic response to therapy [35]. The combination of pegylated IFN and thymosin- $\alpha 1$ is now being evaluated in large phase II/III studies of cirrhotic and noncirrhotic patients who have not responded to previous therapy.

\section{Histamine}

Histamine dihydrochloride acts via $\mathrm{H}_{2}$-type histamine receptors to inhibit the production of oxygen radicals by phagocytes and therefore protects NK cells and T cells from functional inhibition and apoptosis. This mechanism may be important in enhancing the immune response when histamine is combined with IFN. In treatment-naïve patients given histamine dihydrochloride and IFN- $\alpha 2$ b, an overall SVR of $31 \%$ to $38 \%$ was achieved, and in genotype 1 patients response rates of $18 \%$ to $42 \%$ were reported [36]. The drug was tolerated well and is being evaluated in combination with pegylated IFN and ribavirin in phase II studies.

\section{Amantadine}

Amantadine is a broad-spectrum antiviral agent that has been used primarily for treatment of the influenza $A$ virus. Amantadine monotherapy has little effect on $\mathrm{HCV}$ viral levels, but when it is combined with IFN, SVR rates are higher than those with IFN monotherapy in the majority of studies in previously untreated patients. In a recent randomized trial, a beneficial on-treatment response, but not a sustained response, in genotype 1 patients was observed in those who received IFN, ribavirin, and amantadine [37]. However, in another study of patients who had previously not responded to standard therapy, triple therapy was associated with a low SVR rate of $13 \%$ [38].

\section{Molecular-based Strategies}

Specific nonstructural proteins encode several key enzymes involved in HCV viral replication and are an exciting development as potential specific viral drug targets (Fig. 2).

\section{NS2/3, NS3 protease, and NS3 helicase inhibitors}

NS2/3 proteases cleave the nonstructural proteins between NS2 and NS3 polypeptides into functional proteins. It has been proposed that inhibitors of these proteases will target viral replication. The structure of these complex proteins has been defined by radiographic crystallography, allowing characterization of the contact points between proteases and proteins. These contact regions are potential binding sites for protease inhibitors. NS3 serine protease inhibitors, such as BILN 2061, have shown inhibition of HCV RNA in the replicon cell model system, and a recent phase I trial demonstrated a greater than $2-\log ^{10}$ decline in HCV RNA after four doses in most patients [39]. Many other protease inhibitors are in the early phase of development, and longterm studies of safety and efficacy are awaited prior to their recommendation for routine use. However, with prolonged use of these agents, viral resistance is almost certain to occur. Effective use of these agents will probably be best achieved by a combination of inhibitors of different classes, combination with current therapies, or identification of specific inhibitors that evade viral resistance [40].

Helicase enzymes unwind regions of double-stranded RNA or DNA during viral replication or transcription. NS3 helicase inhibitors could prevent the separation of duplex nucleotides that are important in viral replication. These compounds have a complex crystalline structure with mul- 
tiple unique clefts that may be exploited as drug target sites in future classes of helicase inhibitors [41].

\section{Polymerase inhibitors}

HCV viral RNA-dependent RNA polymerase (RdRp) is the product of the NS5B protein. This enzyme is required for the synthesis of negative and positive stranded RNA intermediates necessary for viral replication. The HCV RdRp exhibits the classic "finger-palm-thumb" motif displayed in other nucleic acid polymerases and provides numerous sites that may be targeted by specific polymerase inhibitors. Both nucleoside and non-nucleoside inhibitors of HCV polymerase have been identified primarily through the use of replicon systems. The benzo-1,2,4-thiadiazine derivatives were shown to be highly selective for HCV RNA polymerase and do not appear to inhibit other viral and mammalian polymerases. Non-nucleoside inhibitors, such as JTK-003, have been shown to inhibit conformational change necessary for RNA elongation. This compound was tolerated well in phase I dose-ranging studies and is currently being evaluated in larger-scale trials in patients with HCV [42].

\section{Antisense oligonucleotides}

The HCV IRES has been defined using nuclear magnetic resonance, radiographic crystallography, and cryo-electron microscopy. It binds directly to the $40 \mathrm{~S}$ ribosome subunit, which results in conformational changes within the ribosome. A polyprotein found within the IRES structure functions as a start codon. The conserved HCV IRES is a likely target for effective therapeutic intervention. Extensive efforts have been devoted to the development of antisense oligonucleotides, which are short DNA or RNA sequences specifically designed to bind target mRNA inhibitors targeting HCV IRES sequences that are accessible for nucleic acid hybridization [43]. ISIS 14803 (ISIS Pharmaceuticals, Carlsbad, CA), an oligonucleotide that is complementary to the IRES initiation codon, has been evaluated in phase I and II trials in patients who had not responded to IFNbased therapies [44]. The drug was tolerated well and resulted in a greater than $1-\log ^{10}$ unit reduction in HCV RNA in some patients. However, transient alanine aminotransferase (ALT) flares, though not associated with clinical symptoms, were observed in temporal association with the reduction of plasma HCV RNA in some patients, and also in patients who had not exhibited changes in HCV RNA. Phase II trials are ongoing, and longer-term studies will determine the safety, efficacy, and resistance profile of these approaches [45].

\section{Ribozymes}

Ribozymes are catalytic RNA binders, entailing a catalytic core with two binding arms, that cleave specific RNA sequences. They represent a new potential class of therapeutic agents. Hepatazyme (Ribozyme Pharmaceuticals, Boulder, $\mathrm{CO}$ ), a synthetic first-generation ribozyme prod- uct, has been chemically modified to resist enzymatic and chemical degradation. In its evaluation in replicon systems it was shown to selectively "cut" HCV RNA, thereby inhibiting viral replication [46•]. In phase II trials, results indicate a significant reduction in HCV RNA in approximately $10 \%$ of patients, but toxicities reported in primates have halted further development of this agent. Second-generation ribozymes with improved stability and specificity are in development.

\section{Small interfering RNA compounds}

Small interfering RNAs (siRNAs) are a new potential class of drug targets that efficiently inhibit gene expression by destruction of specific mRNAs [47]. The siRNAs were designed to target the 5'UTR of the HCV genome, which has an internal ribosomal entry site for translation of the entire viral polyprotein. Moreover, the 5'UTR is the most conserved region in the HCV genome, making it an ideal target for these agents. Using a selectable subgenomic HCV replicon cell-culture system, synthetic and vector-derived siRNAs have been shown to specifically inhibit HCV RNA replication and protein expression in Huh-7 cells that consistently replicate the HCV genome, an antiviral effect independent of IFN [48•]. These results suggest that RNAbased gene therapy may represent a new approach to treatment of persistent HCV infection.

\section{P7 inhibitors}

HCV P7 was shown recently to be a polytopic membrane protein that forms ion channels in lipid membranes. Subgenomic replicon studies have shown that the $\mathrm{P} 7$ of $\mathrm{HCV}$ is not necessary for genome replication, and its role in infectious virus production remains unknown. However, these proteins mediate cation permeability across membranes and are important for virion release or maturation. HCV P7 ion channels are inhibited by long-alkyl-chain iminosugar derivatives, which have antiviral activity against the HCV surrogate bovine viral diarrhea virus [49], and recently amantadine was shown to inhibit these channels in an influenza virus model [50]. HCV P7 represents a potential target for antiviral therapy.

\section{Therapies That Modulate \\ Host Immune Responses}

The pathogenesis of chronic HCV infection occurs in the context of an immune response that is inadequate to clear the virus. The Th1 cellular immune response is the dominant response within the liver of HCV-infected patients, producing antiviral cytokines such as IL-2, IFN- $\gamma$, and tumor necrosis factor (TNF)- $\alpha$, which are ineffective in eradicating infection but nevertheless result in hepatic injury. Whereas the intrahepatic cytokine milieu favors a Th1 profile, Th2 cytokines, such as IL-4, IL-5, and IL-10, predominate in the periphery and are associated with extrahepatic manifestations of HCV infection [51]. High 
mutation rates of HCV, which produce escape mutants, probably limit the ability of humoral and cellular responses to clear the virus. Neutralizing antibodies to viral epitopes, which interfere with viral binding to cellular receptors and induce conformational change in viral structure proteins, have recently been identified [52]. Modulation of the host immune response to infection may include enhancement of the cellular immune response, use of therapeutic vaccines, and hyperimmune anti-HCV immunoglobulins.

\section{Anti-HCV immunoglobulin}

The passive transfer of hyperimmune immunoglobulins has been successfully used to prevent or modify hepatitis $\mathrm{A}$ and B infections. A preparation of HCV Ig (HCIg, Civacir; NABI, Rockville, MD) containing broadly cross-reactive antibodies against different HCV strains has been evaluated in chimpanzees. Results from phase I and II studies indicate a decreased rate of HCV replication and a reduction of ALT in chronic infection in two of three chimpanzees [53]. In acute infection, multiple infusions of HCV Ig were associated with a shortened duration of HCV viremia [54]. The method by which HCV replication is affected in this model is yet to be determined. A recent pilot study of 26 patients suggests that HCV Ig does not prevent recurrence of HCV after liver transplantation [55]. Larger studies are required to evaluate the use of HCV Ig alone or in combination with antiviral drugs to decrease peritransplant viremia.

\section{Therapeutic vaccines}

Lack of a cell culture assay for HCV has limited the study of virus neutralization. In addition, the presence of multiple genotypes and quasispecies with a low level of homology within an infected individual has been an obstacle to the identification of highly conserved neutralizing epitopes. Chronic HCV infection is associated with a weak $\mathrm{CD} 4^{+}$and $\mathrm{CD} 8^{+} \mathrm{T}$-cell response. In a phase I study, vaccination with envelope glycoprotein subunit gpE1 successfully stimulated the humoral and cellular immune responses [56]. In a phase IIa study, vaccination with recombinant E1 resulted in a strong E1-specific T-cell response and a significant decline in ALT. Furthermore, patients who demonstrated an increase in E1 showed improvement in liver histology fibrosis scores. Despite the improvement in liver histology, HCV RNA remained unchanged [57]. Longerterm studies to confirm these results and examine diseasemodifying effects are required.

\section{Therapies to Reduce Hepatic Fibrosis}

Chronic hepatitis $\mathrm{C}$ infection is complicated by development of hepatic fibrosis, progression to cirrhosis, and in some patients by progression to hepatocellular carcinoma. The primary aim of therapy is to clear $\mathrm{HCV}$, which is the most effective way to prevent the development of hepatic fibrosis. However, for patients who have established fibro- sis and who have failed to respond to antiviral therapy, additional approaches are required. Unequivocal evidence indicates that HCV-related fibrosis and cirrhosis are reversible, and therapies that can halt or reverse fibrosis are awaited. Stellate cell activation plays a pivotal role in fibrogenesis and is an important future target of antifibrotic therapy. This therapeutic model involves several approaches, including cure of the primary viral disease, inhibition of stellate cell activation, neutralization of stellate cell proinflammatory responses, stimulation of stellate cell apoptosis, and increasing degradation of scar matrix. Numerous compounds are being studied in animal models, but few have been tried in patients with CHC.

The cytokines IFN- $\gamma$ and hepatocyte growth factor have inhibitory effects on stellate cell activation in animal models [58]. IFN- $\gamma$ is being evaluated in a phase II trial in CHC patients with advanced fibrosis. Compounds that downregulate stellate cell activation, such as peroxisome proliferator activated nuclear receptor (PPAR)- $\gamma$ ligands, have also generated interest. These thiazolidinediones prevented early-phase hepatic fibrogenesis in animal models [59] and are currently being evaluated as antifibrotic agents in patients with nonalcoholic steatohepatitis. A significant reduction in fibrosis demonstrated in these cohorts will promote further studies in patients with CHC. Other agents, including vitamin $\mathrm{E}$, hepatocyte growth factor inhibitor, and herbal therapies derived from natural compounds, are also under investigation.

Proliferative cytokine inhibitors, such as plateletderived growth factor antagonist and tissue growth factor (TGF)- $\alpha$ antagonists, are undergoing clinical evaluation. TGF- $\beta$ antagonists, in particular, have the advantage of potentially stimulating scar degradation [60].

\section{Conclusions}

Since the identification of the hepatitis virus and determination of the HCV genome over a decade ago, considerable progress has been made in defining the natural history of HCV infection, understanding its molecular virology, and treating HCV patients. However, a number of challenges remain for the development of more effective antiviral drugs for HCV. Because of the high genetic diversity and geographic distribution of HCV genotypes, antiviral therapy must be effective against different genotypes. In addition, the high rate of mutational change within the HCV genome will inevitably result in drug resistance. Therefore, combination therapy, especially therapy targeting $\mathrm{HCV}$ functional proteins at a variety of sites, is needed. Immunebased therapies with the potential to reverse HCV-induced liver damage, including fibrosis, are another focus for future research. Reproducible and efficient HCV cell-culture systems and small animal models are required for development of these agents.

Optimization of IFN-based regimens for different HCV populations is ongoing. However, with the number of 
promising therapies already in clinical trials, a more effective combination of agents will be available in future years.

\section{References and Recommended Reading}

Papers of particular interest, published recently, have been highlighted as:

- Of importance

-• Of major importance

1. World Health Organization: Hepatitis C: global prevalence (update). Wkly Epidemiol Rec 1999, 49:425-427.

2. Cooreman MP, Schoondermark-Van De Ven EM: Hepatitis C virus: biological and clinical consequences of genetic heterogeneity. Scand J Gastroenterol 1996, 218 (Suppl):106-115.

3. Di Bisceglie AM: Natural history of hepatitis C: its impact on clinical management. Hepatology 2000, 31:1014-1018.

4. Khan M, Farrell G, Byth K, et al.: Which patients with hepatitis C develop liver complications? Hepatology 2000, 31:513-520.

5. Detre KM, Belle SH, Lombardero M: Liver transplantation for chronic viral hepatitis. Viral Hepat Rev 1997, 2:219-228.

6. Simmonds P, Alberti A, Alter HJ, et al.: Second international conference of $\mathrm{HCV}$ and related viruses. Hepatology 1994, 19:1321-1324.

7. Grakoui A, Mccourt DW, Wychowski C, et al.: Characterization of the hepatitis $\mathrm{C}$ virus-encoded serine proteinase: determination of proteinase-dependent polyprotein cleavage sites. $J$ Virol 1993, 67:2832-2843.

8. Gwack Y, Kim DW, Han JH: Characterization of RNA binding activity and RNA helicase activity of the hepatitis C virus NS3 protein. Biochem Biophys Res Commun 1996, 225:654-659.

9. Hwang LH, Hsieh CL, Yen A, et al.: Involvement of the 5' proximal coding sequences of hepatitis $C$ virus with internal initiation of viral translation. Biochem Biophys Res Commun 1998, 252:455-460

$10 . \bullet$ McHutchison JG, Gordon SC, Schiff ER, et al.: Interferon alfa-2b alone or in combination with ribavirin as initial treatment for chronic hepatitis C. N Engl J Med

1998, 339:1485-1492.

A multicenter trial demonstrating that initial therapy with interferon and ribavirin was more effective than treatment with interferon alone.

$11 . \bullet$ Poynard T, Marcellin P, Lee SS, et al.: Randomised trial of interferon alpha-2b plus ribavirin for 48 weeks or for 24 weeks versus interferon alpha-2b plus placebo for 48 weeks for treatment of chronic infection with hepatitis $\mathrm{C}$ virus. Lancet 1998, 352:1426-1432.

A multicenter trial demonstrating that the combination of interferon and ribavirin is more effective than 48 weeks of interferon monotherapy. Patients with few favorable factors benefited more from extending the duration of combination therapy to 48 weeks.

12.• Manns MP, Mchutchison JG, Gordon SC, et al.: Peginterferon alfa-2b plus ribavirin compared with interferon alfa- $2 b$ plus ribavirin for initial treatment of chronic hepatitis C: a randomised trial. Lancet 2001, 358:958-965.

A multicenter trial demonstrating that combination of pegylated interferon and ribavirin is more effective than standard interferon combination therapy. The benefit was mostly achieved in patients with HCV genotype 1 infection.

13.•• Fried MW, Shiffman ML, Reddy KR, et al.: Peginterferon alfa-2a plus ribavirin for chronic hepatitis $\mathrm{C}$ virus infection. $\mathrm{N}$ Engl J Med 2002, 347:975-982.

A multicenter trial demonstrating that the combination of pegylated interferon and ribavirin is more effective than standard interferon combination therapy and pegylated interferon monotherapy.

14. Bukh J, Forns X, Emerson SU, et al.: Studies of hepatitis C virus in chimpanzees and their importance for vaccine development. Intervirology 2001, 44:132-142.

15. Ilan E, Arazi J, Nussbaum $\mathrm{O}$, et al.: The hepatitis $\mathrm{C}$ virus (HCV)-Trimera mouse: a model for evaluation of agents against HCV. J Infect Dis 2002, 185:153-161.
16. Mercer DF, Schiller DE, Elliott JF, et al.: Hepatitis C virus replication in mice with chimeric human livers. Nat Med 2001, 7:927-933.

17. Lohmann V, Korner F, Koch J, et al.: Replication of subgenomic hepatitis $\mathrm{C}$ virus RNAs in a hepatoma cell line. Science 1999, 285:110-113.

18. Castet V, Fournier C, Soulier A, et al.: Alpha interferon inhibits hepatitis $\mathrm{C}$ virus replication in primary human hepatocytes infected in vitro. $J$ Virol 2002, 76:8189-8199.

19. Tomassini JE, Boots $\mathrm{E}$, Gan $\mathrm{L}$, et al.: An in vitro Flaviviridae replicase system capable of authentic RNA replication. Virology 2003, 313:274-285.

20. Zein NN: Interferons in the management of viral hepatitis. Cytokines Cell Mol Ther 1998, 4:229-241.

21. Tong MJ, Blatt LM, Resser KJ, et al.: Treatment of chronic hepatitis $\mathrm{C}$ virus infection with recombinant consensus interferon. J Interferon Cytokine Res 1998, 18:81-86.

22. Haydon GH, Mutimer DJ: Genetically modified interferon: Is there a consensus yet? Eur J Gastroenterol Hepatol 2002, 14:471-473.

23. Chang CC, Chen TT, Cox BW, et al.: Evolution of a cytokine using DNA family shuffling. Nat Biotechnol 1999, 17:793-797.

24. Osborn BL, Olsen HS, Nardelli B, et al.: Pharmacokinetic and pharmacodynamic studies of a human serum albumin-interferon-alpha fusion protein in cynomolgus monkeys. J Pharmacol Exp Ther 2002, 303:540-548.

25. Frese $M$, Schwarzle V, Barth $K$, et al.: Interferon-gamma inhibits replication of subgenomic and genomic hepatitis $\mathrm{C}$ virus RNAs. Hepatology 2002, 35:694-703.

26. Tompkins WA: Immunomodulation and therapeutic effects of the oral use of interferon-alpha: mechanism of action. J Interferon Cytokine Res 1999, 19:817-828.

27. Eklind J, Tartler U, Maschke J, et al.: Imiquimod to treat different cancers of the epidermis. Dermatol Surg 2003, 29:890-896

28. Zhang Y, Jamaluddin M, Wang S, et al.: Ribavirin treatment upregulates antiviral gene expression via the interferon-stimulated response element in respiratory syncytial virus-infected epithelial cells. J Virol 2003, 77:5933-5947.

Ribavirin potentiates activity of interferon-signaling pathways,

suggesting a mechanism for the efficacy of combined interferon and ribavirin therapy.

29. Watson J: Prospects for hepatitis c virus therapeutics: levovirin and viramidine improved derivatives of ribavirin. Curr Opin Investig Drugs 2002, 3:680-683.

30. Tam RC, Ramasamy K, Bard J, et al.: The ribavirin analog ICN 17261 demonstrates reduced toxicity and antiviral effects with retention of both immunomodulatory activity and reduction of hepatitis-induced serum alanine aminotransferase levels. Antimicrob Agents Chemother 2000, 44:1276-1283.

31. Arora S, Lau D, Gish R, et al.: Phase I clinical studies of viramidine: a liver-targeting prodrug of ribavirin. Hepatology 2002, 36:356A.

32. McHutchison JG, Cheung R, Shiffman ML, et al.: A 4-week trial of VX 497 (an IMPDH inhibitor) combined with interferon in previously untreated patients with chronic hepatitis $\mathrm{C}$. Hepatology 2001, 34(Suppl):329A

33. Cornberg M, Hinrichsen $\mathrm{H}$, Teuber $\mathrm{G}$, et al.: Mycophenolate mofetil in combination with recombinant interferon alfa-2a in interferon-nonresponder patients with chronic hepatitis C. J Hepatol 2002, 37:843-847.

34. Afdhal N, Flamm S, Imperial JC, et al.: Analyses of $40 \mathrm{KDA}$ peginterferon alfa-2a in combination with ribavirin, mycophenolate mofetil, amantadine or amantadine plus ribavirin in patients that relapsed or did not respond to Rebetron therapy: a report of two randomized, multicenter, efficacy and safety studies. Hepatology 2001, 34(Suppl):243A.

35. Pockros PJ: Developments in treatment of chronic hepatitis C. Expert Opin Investig Drugs 2002, 11:1-14. 
36. Lurie $Y$, Nevens F, Aprosina ZG, et al.: A multicentre, randomized study to evaluate the safety and efficacy of histamine dihydrochloride and interferon-alpha-2b for the treatment of chronic hepatitis C. J Viral Hepat 2002, 9:346-353.

37. Berg $\mathrm{T}$, Kronenberger B, Hinrichsen $\mathrm{H}$, et al.: Triple therapy with amantadine in treatment-naive patients with chronic hepatitis C: a placebo-controlled trial. Hepatology 2003, 37:1359-1367.

38. Thuluvath Pj, Pande H, Maygers J: Combination therapy with interferon-alpha(2b), ribavirin, and amantadine in chronic hepatitis $\mathrm{C}$ nonresponders to interferon and ribavirin. Dig Dis Sci 2003, 48:594-597.

39. Hinrichsen $H$, Benhamou $Y$, Reiser $M$, et al.: First report on the antiviral efficacy of BILN 2061, a novel oral HCV serine proteases inhibitor, in patients with chronic hepatitis c genotype 1. Hepatology 2002, 36(Suppl 2):866A.

40. Casbarra A, Piaz FD, Ingallinella P, et al.: The effect of prime-site occupancy on the hepatitis C virus NS3 protease structure. Protein Sci 2002, 11:2102-2112.

41. Myles DC: Recent advances in the discovery of small molecule therapies for HCV. Curr Opin Drug Discov Devel 2001, 4:411-416.

42. Walker M, Hong Z: HCV-RNA dependent RNA polymerase as a target for anti-viral development. Curr Opin Pharmacol 2002, 2:534-540.

43. Zhang H, Hanecak R, Brown-Diver V, et al.: Antisense oligonucleotide inhibition of HCV gene expression in livers of mice infected with an HCV-vaccinia virus recombinant. Antimicrob Agents Chemother 1999, 43:347-353.

44. Mchutchison J, Pockros P, Patel K, et al.: A phase 1b dose escalation trial of ISIS 14803, an antisense inhibitor of HCV, in patients with chronic HCV: final report. Hepatology 2002, 36(Suppl 2):303A.

45. Gordon SC, Bacon BR, Jacobson IM, et al.: A phase II, $\mathbf{1 2}$ week study of ISIS 14803, an antisense inhibitor of HCV for the treatment of chronic HCV. Hepatology 2002, 36(Suppl 2):362A.

46. Macejak DG, Jensen KL, Jamison SF, et al.: Inhibition of hepatitis $\mathrm{C}$ virus (HCV)-RNA-dependent translation and replication of a chimeric HCV poliovirus using synthetic stabilized ribozymes. Hepatology 2000, 31:769-776.

Activity of ribozymes targeting site 195 of HCV RNA exhibits a significant inhibition of HCV replication, demonstrating potential antiviral benefits.

47. Hannon GJ: RNA interference. Nature 2002, 418:244-251.
48. Kapadia SB, Brideau-Andersen A, Chisari FV: Interference of hepatitis $\mathrm{C}$ virus RNA replication by short interfering RNAs. Proc Natl Acad Sci U S A 2003, 100:2014-2018.

RNA can specifically inhibit HCV RNA replication in Huh-7 cells that stably replicate the HCV genome and may represent a new approach for the treatment of persistent $\mathrm{HCV}$ infection.

49. Pavlovic D, Neville DC, Argaud O, et al:: The hepatitis $\mathrm{C}$ virus p7 protein forms an ion channel that is inhibited by longalkyl-chain iminosugar derivatives. Proc Natl Acad Sci U S A 2003, 100:6104-6108.

50. Griffin SD, Beales LP, Clarke DS, et al:: The $\mathbf{p} 7$ protein of hepatitis $\mathrm{C}$ virus forms an ion channel that is blocked by the antiviral drug, amantadine. FEBS Lett 2003, 535:34-38.

51. Chen $M$, Sallberg $M$, Sonnerborg A, et al.: Limited humoral immunity in hepatitis $\mathrm{C}$ virus infection. Gastroenterology 1999, 116:135-143.

52. Takikawa S, Ishii $\mathrm{K}$, Aizaki $\mathrm{H}$, et al.: Cell fusion activity of hepatitis C virus envelope proteins. J Virol 2000, 74:5066-5074.

53. Krawczynski K, Fattom A, Spelbring J, et al.: Early termination of HCV infection by passive anti-HCV transfer in experimentally infected chimpanzees. Hepatology 1998, 28:398A.

54. Krawczynski K, Fattom A, Culver D, et al.: Passive transfer of anti-HCV in chronic and acute HCV infection in chimpanzees: trials of experimental immune treatment. Hepatology 1999, 30:423A.

55. Willems B, Ede M, Marotta P, et al:: Anti-HCV human immunoglobulins for the prevention of graft infection in HCV-related liver transplantation: a pilot study. J Hepatol 2002, 36:S32.

56. Leroux-Roels G, Depla E, Hulstaert F, et al.: A candidate therapeutic vaccine for chronic hepatitis $\mathrm{C}$ infection based on envelope 1 protein: tolerability and immunogenicity in healthy adult volunteers. Hepatology 2000, 34:449A.

57. Maertens G: Therapeutic Vaccines. Presented at the Hepatitis C Single Topic Conference. February 27-March 1 2003, Chicago, IL.

58. Ziesche R, Hofbauer E, Wittmann $\mathrm{K}$, et al.: A preliminary study of long-term treatment with interferon gamma-1b and lowdose prednisolone in patients with idiopathic pulmonary fibrosis. N Engl J Med 1999, 341:1264-1269.

59. Galli A, Crabb DW, Ceni E: Antidiabetic thiazolidinediones inhibit collagen synthesis and hepatic stellate cell activation in vivo and in vitro. Gastroenterology 2002, 122:1924-1940.

60. George J, Roulot D, Koteliansky VE, Bissell DM: In vivo inhibition of rat stellate cell activation by soluble transforming growth factor beta type II receptor: a potential new therapy for hepatic fibrosis. Proc Natl Acad Sci U S A 1999, 96:12719-12724.

61. Davis GL, Nelson DR, Reyes GR: Future options for the management of hepatitis C. Semin Liver Dis 1999, 19:103-112. 